\title{
A commentary on the gender-specific association of TSNAX/DISC1 locus for schizophrenia and bipolar affective disorder in South Indian population
}

\author{
Shusuke Numata \\ Journal of Human Genetics (2012) 57, 475-476; doi:10.1038/jhg.2012.82; published online 5 July 2012
}

$\mathrm{T}$ The disrupted-in-schizophrenia-1 (DISC1) gene was originally identified from the breakpoint of a chromosomal translocation $(1 ; 11)(\mathrm{q} 42.1 ; \mathrm{q} 14.3)$ in a large Scottish family with major mental disorders, including schizophrenia (SCZ), bipolar affective disorder (BPAD) and recurrent major depression. ${ }^{1,2}$ Subsequently, many genetic evidences for the involvement of DISC1 in SCZ have been reported in different populations, ${ }^{3}$ and DISC1 has become one of the putative susceptibility genes for SCZ. In this issue, Ram Murthy et al. ${ }^{4}$ reported a genderspecific DISC1 contribution in SCZ, BPAD and combined samples in South Indian population.

The first concern is the risk alleles and the haplotypes identified. Although the results of genetic replication studies between SCZ and the DISC1 gene must be interpreted with extreme caution, ${ }^{5}$ a critical issue in positive DISC1 association studies is a lack of replication for the same alleles in the same direction and the same haplotypes across the studies. In addition, recent genome-wide association studies (GWAS) $)^{6,7}$ as well as a large association study ${ }^{8}$ and a meta-analysis of variants in the DISC1 gene $^{9}$ failed to replicate significant associations of the DISC1 gene with SCZ. The second concern is a gender-specific association of DISC1 in SCZ. A meta-analysis of published literature has demonstrated a sex difference in the risk of developing SCZ, ${ }^{10}$ and several studies have demonstrated sex-specific associations of

Dr S Numata is at the Department of Psychiatry, Course of Integrated Brain Sciences, Medical Informatics, Institute of Health Biosciences, The University of Tokushima Graduate School, Tokushima, Japan.

E-mail: shu-numata@umin.ac.jp
DISC1 in SCZ. ${ }^{3}$ However, the GWAS has produced evidence that the genetic basis of SCZ is the same in males and females. ${ }^{11}$ When the data are subdivided on the basis of sex in genetic association studies, one major problem that arises is the lack or reduction of power, and another is to claim nominal statistical significance, which may be occurred by chance. The sample size of the present study was small, and most of their sex-specific findings did not remain significant after correction for multiple comparisons. ${ }^{4}$ Patsopoulos et al. ${ }^{12}$ empirically evaluated observational 432 studies claiming to have found sex-related differences in genetic effects for common diseases and traits, and demonstrated that the majority of these claims were insufficiently documented or spurious. The third concern is a genetic overlap between SCZ and BPAD in the DISC1 gene. As described above, the translocation event involving DISC1 increases the risk of both SCZ and BPAD. ${ }^{1,2}$ Lichtenstein et al. ${ }^{13}$ reported that first-degree relatives of probands with either SCZ or BPD were at increased risk of these disorders, and halfsiblings had an increased risk, but lower than that of the full-siblings, suggesting that SCZ and BPD partly share a common genetic cause. Similar to this epidemiological study, the GWAS revealed that common polygenic variation contributed to the risk of SCZ and BPD. ${ }^{6}$ From this point of view, the present study's finding of a genetic overlap between SCZ and BPAD in the DISC1 gene ${ }^{4}$ is not surprising.

In conclusion, the majority of previous genetic association studies of SCZ, including GWAS, have been underpowered to detect common risk alleles with small effect sizes. To determine whether common DISC1 genetic variants are truly implicated in SCZ, association studies using large samples and metaanalyses of previous literature are essential. Recent GWAS analyses of large collaborative samples have been remarkably successful for other common diseases, such as Crohn's disease and type 2 diabetes. For psychiatric disorders, the Psychiatric Genomewide Association Study Consortium (PGC) was organized. PGC is extending its samples and is performing large scale collaborative analyses using both traditional disorder categories and non-traditional analyses that cut across diagnostic categories (cross-disorder analyses). ${ }^{6,7}$ It is still difficult to come to a conclusion of the involvement of common DISC1 variants in SCZ in the general population, and there is a possibility that other mechanisms, such as copy number variants, epigenetics (histone and DNA modifications) and microRNAs, could explain the involvement of DISC1 in SCZ in the future. Dr Sawa, ${ }^{14}$ one of the most distinguished DISC1 researchers, said that the molecular pathways involving DISC1 seem to be implicated in unique endophenotypes that underlie many distinct mental disorders labelled by current diagnostic manuals, thus, DISC1 biology may possibly provide a hint for constructing new diagnostic criteria for mental disorders at a more biological, and probably more relevant.

\footnotetext{
1 St Clair, D., Blackwood, D., Muir, W., Carothers, A., Walker, M., Spowart, G. et al. Association within a family of a balanced autosomal translocation with major mental illness. Lancet 336, 13-16 (1990).
} 
2 Millar, J. K., Wilson-Annan, J. C., Anderson, S., Christie, S., Taylor, M. S., Semple, C. A. et al. Disruption of two novel genes by a translocation co-segregating with schizophrenia. Hum. Mol. Genet. 9, 1415-1423 (2000).

3 Chubb, J. E., Bradshaw, N. J., Soares, D. C., Porteous, D. J. \& Millar, J. K. The DISC locus in psychiatric illness. Mol. Psychiatry 13, 36-64 (2008).

4 Ram Murthy, A., Purushottam, M., Kiran Kumar, H. B., ValliKiran, M., Krishna, N., Sriharsha, K. J. et al. Gender specific association of TSNAX/DISC1 locus for schizophrenia and bipolar affective disorder in South Indian population. J. Hum. Genet. 57, 523-530 (2012).

5 Sullivan, P. F. Spurious genetic associations. Biol. Psychiatry 61, 1121-1126 (2007).

6 Purcell, S. M., Wray, N. R., Stone, J. L., Visscher, P. M., O'Donovan, M. C., Sullivan, P. F. et al. Common polygenic variation contributes to risk of schizophrenia and bipolar disorder. Nature 460, 748-752 (2009).

7 Ripke, S., Sanders, A. R., Kendler, K. S., Levinson, D. F., Sklar, P., Holmans, P. A. et al. Genome-wide association study identifies five new schizophrenia loci. Nat. Genet. 43, 969-976 (2011).

8 Sanders, A. R., Duan, J., Levinson, D. F., Shi, J., He D., Hou, C. et al. No significant association of 14 candidate genes with schizophrenia in a large European ancestry sample: implications for psychiatric genetics. Am. J. Psychiatry 165, 497-506 (2008).

9 Mathieson, I., Munafò, M. R. \& Flint, J. Meta-analysis indicates that common variants at the DISC1 locus are not associated with schizophrenia. Mol. Psychiatry 17, 634-641 (2012).

10 Aleman, A., Kahn, R. S. \& Selten, J. P. Sex differences in the risk of schizophrenia: evidence from metaanalysis. Arch. Gen. Psychiatry 60, 565-571 (2003).
11 Lee, S. H., DeCandia, T. R., Ripke, S., Yang, J., Sullivan, P. F., Goddard, M. E. et al. Estimating the proportion of variation in susceptibility to schizophrenia captured by common SNPs. Nat. Genet. 44 247-250 (2012).

12 Patsopoulos, N. A., Tatsioni, A. \& loannidis, J. P. Claims of sex differences: an empirical assessment in genetic associations. JAMA 298, 880-893 (2007).

13 Lichtenstein, P., Yip, B. H., Björk, C., Pawitan, Y. Cannon, T. D., Sullivan, P. F. et al. Common genetic determinants of schizophrenia and bipolar disorder in Swedish families: a population-based study. Lancet 373, 234-239 (2009).

14 Brandon, N.J. \& Sawa, A. Linking neurodevelopmental and synaptic theories of mental illness through DISC1. Nat. Rev. Neurosci. 12, 707-722 (2011). 